\title{
Impfungen und Prävention für Asylbewerber
}

— Wenn Asylbewerber, die bereits in den Kommunen wohnen, krank werden, erhalten sie Leistungen gemäß Asylbewerberleistungsgesetz (AsylbewerberLeistungsGesetz $\S 4$ und 6). Dieses sieht eine Behandlung von Schmerzzuständen oder aku- ten Erkrankungen vor. Die Kosten für die medizinische Behandlung trägt das örtliche Sozialamt. Daher müssen Asylbewerber einen Berechtigungsschein beantragen, der sie persönlich für den Arztbesuch befugt. Pro Quartal gibt es einen Krankenbehand-

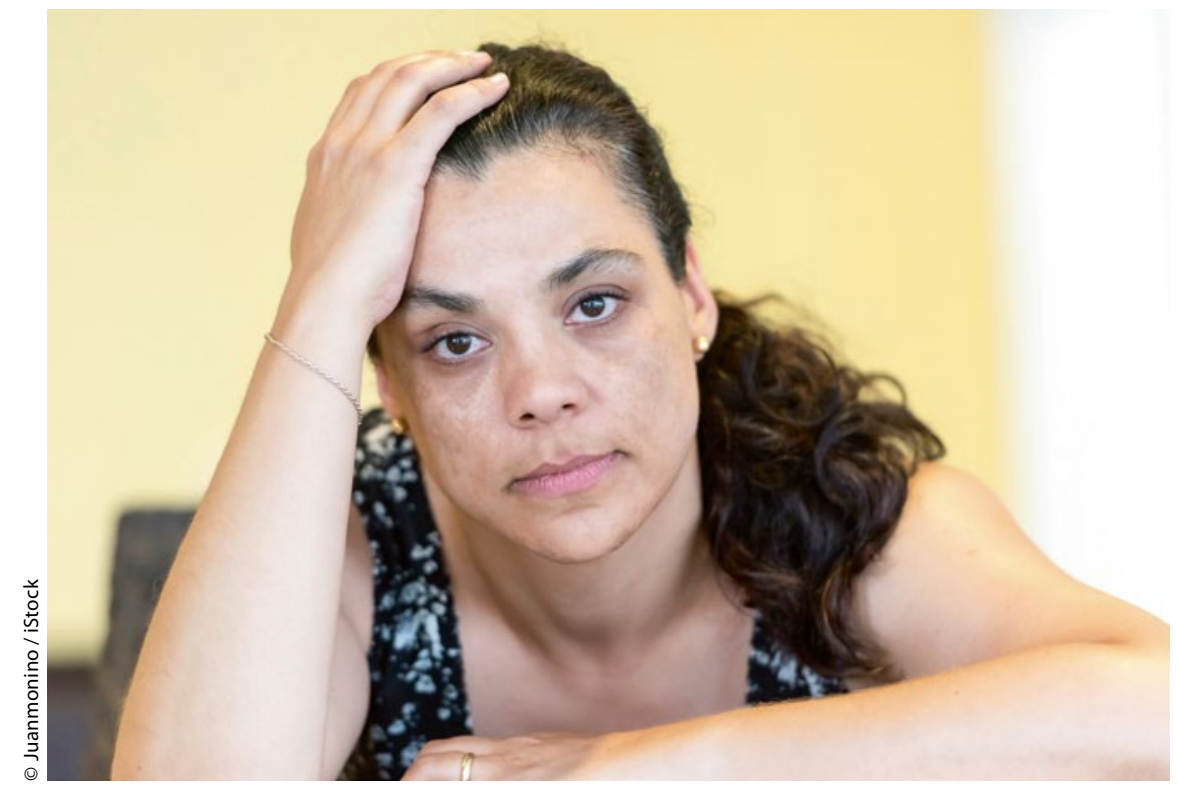

Kranke Asylbewerber müssen sich zunächst mit der Bürokratie herumschlagen. lungsschein, der beim ersten Arztbesuch vorgelegt werden muss. In der Folge dient er auch als Überweisungsschein. In Notfällen kann der Berechtigungsschein auch nachgereicht werden.

\section{MMW-Kommentar}

Die zuständige Behörde übernimmt aber auch die Kosten für amtlich empfohlene Schutzimpfungen und medizinisch gebotene Vorsorgeuntersuchungen. Die Abrechnung erfolgt grundsätzlich über die KV. Asylbewerber sind von Zuzahlungen z. B. bei Arzneimitteln befreit. Die Kosten übernimmt zu 100\% das Sozialamt, wenn die Gültigkeit des Krankenscheines gegeben ist. Auf den Rezepten muss deshalb immer "gebührenfrei" angekreuzt und vermerkt werden, dass mit dem Kostenträger abzurechnen ist. Sollte eine über die gesetzliche Vorschrift hinausgehende Behandlung erforderlich sein (z. B. Krankengymnastik oder ein Hilfsmittel), ist im Einzelfall die Genehmigung des Kostenträgers vorher einzuholen. Jegliche stationäre Behandlung, soweit es kein akuter Notfall ist, bedarf ebenfalls grundsätzlich der vorherigen Genehmigung.

\section{So rechnen Sie Praxis-Urintests richtig ab}

- Als Urinstatus werden in der Praxis üblicherweise eine Teststreifenuntersuchung und die Untersuchung des Sedimentes bezeichnet. Hierzu steht zur Abrechnung die Nr. 32030 EBM (Orientierende Untersuchung mittels vorgefertigter Testträger) zur Verfügung. Diese Leistung ist nur einmal abrechenbar, auch wenn verschiedene Parameter mit unterschiedlichen Teststreifen untersucht werden.

Diese Teststreifenuntersuchung wird aktuell im Rahmen der bundesweit einheitlichen Quotierung von Laborleistungen zu 91,58\% und deshalb nur mit 0,46 Euro vergütet. Die Leistung beinhaltet auch eine eventuelle apparative Auswertung des Streifens. Gemäß der Präambel zum Abschnitt 32.2.1 ist die alleinige Abrechnung einzelner Parameter nicht möglich: „Der Nachweis von Eiweiß und/oder Glukose im Harn (ggf. einschl. Kontrolle auf Ascorbinsäure) sowie die Bestimmung des spezifischen Gewichts und/oder des $\mathrm{pH}$-Wertes im Harn ist nicht berechnungsfähig".

Die Testmaterialien für die Untersuchungen nach Nr. 32030 stellen Praxiskosten dar, lediglich die Materialien für die nicht abrechenbaren Leistungen Eiweiß, Glukose und pH-Wert können über den Sprechstundenbedarf bezogen werden.

\section{MMW-Kommentar}

Die Untersuchung des Urinsediments kann nach Nr. 32031 berechnet werden und bringt nach Quotierung ein Honorar von 0,23 Euro. Die Nrn. 32030 und 32031 sind nicht neben den Leistungen der präoperativen Diagnostik nach den Nrn. 31010 bis 31013 berechnungsfähig, die Nr. 32030 auch nicht neben der Gesundheitsuntersuchung nach Nr. 01732. In diesem Fall kann aber die Nr. 32880 berechnet werden, die Laborpauschale für Untersuchungen im Zusammenhang mit der Gesundheitsuntersuchung unter Nutzung eines Teststreifens. Diese Leistung wird unbudgetiert mit 0,50 Euro vergütet. 